\title{
Desempenho reprodutivo de fêmeas suínas submetidas à inseminação artificial intra- uterina ou à tradicional
}

\author{
Reproductive performance of sows submitted to intrauterine or tradicional artificial insemination
}

\author{
Paulo Eduardo Bennemann ${ }^{\mathrm{I}}$ Felipe Leonardo Koller $^{\mathrm{I}}$ Mari Lourdes Bernardi $^{\mathrm{II}}$ Ivo Wentz \\ Fernando Pandolfo Bortolozzo
}

RESUMO

Foram utilizadas 298 fêmeas pluríparas Camborough $22^{\circledast}$ distribuídas em dois tratamentos: T1 ( $\left.n=154\right)$, inseminação intra-uterina (IAU) com dose inseminante (DI) contendo 0,5 bilhão de espermatozóides em volume total de 20ml; e T2 ( $n=144)$, inseminação tradicional (IAT), com DI contendo 3,0 bilhões de espermatozóides em volume total de $90 \mathrm{ml}$. Foi possível a realização da IAU em 98,1\% das fêmeas. A presença de sangue na extremidade do cateter ou espiral da pipeta de IAU foi observada em 8,4\% das fêmeas. As taxas de prenhez (TPr) e de parto ajustada não diferiram $(P>0,05)$ entre a IAU e IAT. O tamanho da leitegada (TL) diferiu entre os tratamentos $(P<0,05)$, sendo observada redução de 0,8 leitão na IAU. A presença de sangue na IAU não afetou a TPr significativamente, mas reduziu o TL em 2,6 leitões.

Palavras-chave: inseminação intra-uterina, inseminação artificial, suínos.

\section{ABSTRACT}

A total of 298 Camborough $22^{\circledR}$ sows was distributed in two treatments: $T 1(n=154)$ : intrauterine insemination (IUI) with 0.5 billion sperms in $20 \mathrm{ml}$ total volume; T2 ( $n=144)$ : traditional insemination (TAI) with 3.0 billion sperms in $90 \mathrm{ml}$ total volume. It was possible to perform the IUI in $98.1 \%$ of sows. It was observed presence of blood on the catheter tip or pipete in $8.4 \%$ of IUI sows. The pregnancy $(P R)$ and adjusted farrowing rates did not differ $(P>0.05)$ among treatments. Litter size (LS) differ among treatments $(P<0.05)$, being observed a reduction of 0.8 piglet in IUI. The blood presence in the IUI did not affect the PR significantly, but resulted in a reduction of 2.6 piglets per litter.

Key words: intrauterine insemination, artificial insemination, swine.

\section{INTRODUÇÃO}

Durante a década de 60, fêmeas suínas foram inseminadas utilizando-se diferentes volumes e número de espermatozóides, sendo observado que eram necessários, no mínimo, $5 \times 10^{9}$ espermatozóides em 100ml para se alcançar bons índices de fecundação (BAKER et al., 1968). Embora HANCOCK (1959) e HANCOCK \& HOVELL (1961) tenham demonstrado que a fertilidade varia de acordo com o local de deposição do sêmen, nos últimos 40 anos o volume e o número de espermatozóides por dose inseminante (DI) foram pouco alterados. Atualmente, na inseminação artificial tradicional (IAT), são utilizadas de duas a três doses inseminantes (DIs) por estro, cada uma delas contendo de dois a quatro bilhões de espermatozóides, diluídos em 80 a 100ml de volume, totalizando até 12 bilhões de espermatozóides por fêmea inseminada (RATH et al., 2000; MARTINEZ et al., 2001).

Nos últimos cinco anos, vários estudos têm confirmado que o número de espermatozóides, bem como o volume da DI, podem ser reduzidos, sem prejuízo à fertilidade, se a deposição do sêmen for realizada cranialmente à cérvix (MARTINEZ et al., 2001; WOLKEN et al., 2002; WATSON \& BEHAN, 2002). Recentemente, foi demonstrado que, com a inseminação intra-uterina (IAU), é possível a utilização de doses com apenas 500 milhões de espermatozóides sem prejuízo à taxa de prenhez e ao número total de embriões (MEZALIRA et al., 2005). No entanto, taxa de parto e número de leitões nascidos com a utilização desta

\footnotetext{
IDepartamento de Medicina Animal - Setor Suínos, Faculdade de Veterinária, Universidade Federal do Rio Grande do Sul (UFRGS), Bento Gonçalves, 9090, 91540-000, Porto Alegre, RS, Brasil. E-mail: fpbortol@ufrgs.br. *Autor para correspondência.

"Departamento de Zootecnia, Faculdade de Agronomia, UFRGS, Porto Alegre, RS, Brasil.
} 
quantidade de espermatozóides por DI ainda são pouco estudados.

O objetivo deste estudo foi avaliar o desempenho reprodutivo, baseado na taxa de prenhez, na taxa de parto ajustada e no tamanho da leitegada, de fêmeas suínas submetidas à técnica de IAU com 0,5 bilhão de espermatozóides, em comparação à IAT com três bilhões de espermatozóides.

\section{MATERIAL E MÉTODOS}

Foram utilizadas 298 fêmeas pluríparas Camborough $22^{\circledR}$ do plantel reprodutivo de uma granja produtora de leitões. As matrizes foram separadas de acordo com a ordem de parto (1-8), a média do tamanho da leitegada dos partos anteriores (7-10, >10-12, >12), o intervalo desmame-estro (2-9), os dias de lactação (12-22) e escore corporal visual (2-5), para serem aleatoriamente distribuídas em dois tratamentos. O tratamento $1(\mathrm{n}=154)$ consistiu de inseminações pela técnica de IAU com doses contendo 0,5 bilhão de espermatozóides em volume total de $20 \mathrm{ml}$. No tratamento $2(\mathrm{n}=144)$, foi efetuada a IAT com doses contendo 3,0 bilhões de espermatozóides em volume total de $90 \mathrm{ml}$.

Após o desmame, as matrizes foram alojadas em gaiolas individuais dotadas de um cocho acessório. $\mathrm{O}$ arraçoamento foi realizado à vontade, com ração lactação (3.300Kcal EM kg-1) até o início do estro. As matrizes que demonstraram sinais de estro foram deslocadas para a linha de cobertura, sendo arraçoadas com ração gestação $\left(2.950 \mathrm{Kcal} \mathrm{EM} \mathrm{kg}^{-1}\right)$. Após a inseminação, as matrizes foram mantidas em regime de restrição alimentar $\left(1,8 \mathrm{~kg} \mathrm{dia}^{-1}\right)$ durante cinco dias.

O diagnóstico do estro foi realizado duas vezes ao dia, às 7h30min e às 15h30min, com auxílio de um macho sexualmente maduro. Foram utilizadas somente as fêmeas que manifestaram estro no turno da manhã. Para o cálculo do início do estro, foi considerado o momento em que a fêmea apresentou reflexo de imobilidade à pressão lombar exercida por um funcionário, na presença do macho, menos oito horas (BENNEMANN et al., 2005). O final do estro foi definido como o momento em que a fêmea não apresentou mais o reflexo de imobilidade à pressão lombar, menos quatro (quando à tarde) ou oito horas (quando pela manhã).

A determinação do momento da ovulação foi realizada por ultra-sonografia transcutânea, com auxílio de um transdutor setorial de cinco MHz (Aloka Co., Ltd., Mure, Mitaka-shi, Tokyo 181-8622, Japan), a cada 12 horas (7h e às 19h), a partir do turno seguinte à manifestação do estro. O momento da ovulação foi definido como o primeiro exame ultra-sonográfico sem a presença de folículos pré-ovulatórios, menos seis horas (BENNEMANN et al., 2005). A ovulação foi confirmada por um exame adicional, 12 horas mais tarde. Como doadores de sêmen, foram utilizados 10 machos adultos Agroceres PIC ${ }^{\circledR}$ mantidos em regime de duas coletas semanais. $\mathrm{O}$ ejaculado foi submetido a exame macro (cor, odor, aspecto e volume) e microscópico (motilidade, concentração, aglutinação e morfologia espermática). Foram processados somente ejaculados que apresentaram no mínimo $75 \%$ de motilidade. A determinação da concentração espermática (espermatozóides/ml) foi realizada por contagem em câmara de Neubauer Improved ${ }^{\circledR}$. Após a diluição, foi realizada nova contagem do número de espermatozóides DI. As DIs foram preparadas a partir da mistura de dois ejaculados por "Split-sample", sendo confeccionadas doses inseminantes com 3,0 e 0,5 bilhões de espermatozóides totais, diluídos em BTS, em volume total de 90 e $20 \mathrm{ml}$, respectivamente. As doses foram armazenadas a temperaturas entre 15 e $18^{\circ} \mathrm{C}$, em período máximo de 30 horas.

A IAT foi realizada com pipeta de Melrose. Para a IAU, foi utilizada uma pipeta de IA descartável Supertip ${ }^{\circledR}$ com cateter modelo Verona ${ }^{\circledR}$ (Minitub). Após a fixação da pipeta na cérvix, o cateter foi introduzido lentamente no lúmen uterino (cerca de 200mm), permitindo, dessa forma, a deposição das células espermáticas em local cranial à cérvix, no ambiente uterino. As matrizes foram inseminadas apenas uma vez ao dia, sendo a primeira inseminação efetuada no turno seguinte à manifestação do estro e as seguintes com intervalos de 24 horas, enquanto a fêmea esteve em estro. No momento da inseminação, foi registrada a presença ou ausência de refluxo de sêmen. Após a IAU, foi realizada a inspeção da pipeta e do cateter e, 120 minutos após, do vestíbulo e da vagina, quanto à presença ou ausência de sangue.

O controle de retorno ao estro foi iniciado a partir do $18^{\circ}$ dia após a IA e, ao $23^{\circ}$ dia, foi realizado o diagnóstico de gestação por ultra-sonografia transcutânea em tempo real. Ao parto, foram coletados os dados do tamanho da leitegada (TL).

O delineamento experimental foi completamente casualizado. As variáveis taxa de prenhez (TPr) e taxa de parto ajustada (TPA), que excluíram as fêmeas mortas e descartadas por problemas não-reprodutivos, foram analisadas pelo teste de Quiquadrado. O TL foi analisado pelo procedimento GLM, sendo as médias calculadas pelo procedimento LSMEANS e comparadas pelo teste $t$ (SAS, 1998). Além do efeito do tratamento, foram testadas no modelo as covariáveis ordem de parto, intervalo desmame-estro, duração da lactação, escore corporal visual e média do 
tamanho da leitegada dos partos anteriores. Apenas a média do tamanho da leitegada dos partos anteriores apresentou efeito significativo e foi mantida no modelo como covariável para TL.

\section{RESULTADOS E DISCUSSÃO}

A duração média do estro foi de $70,3 \pm 17,8$ horas (24 a 144 horas), e o intervalo médio entre o seu início e a ovulação foi de 42,1 $\pm 11,0$ horas (18 a 90 horas). A passagem do cateter através do canal cervical e realização da IAU foi possível em 98,1\% das fêmeas (151/154). Em outros trabalhos(WATSON \& BEHAN, 2002; DALLANORA et al., 2004), nos quais a IAU foi realizada com cateteres semelhantes ao utilizado nesse estudo, o sucesso na passagem pela cérvix foi superior a 95\%. Segundo MARTINEZ et al. (2002), a ordem de parto (2 a 6) das fêmeas não teve influência no grau de dificuldade e no tempo despendido para realizar a IAU. Dessa forma, é possível que características anatômicas, específicas de cada fêmea, sejam responsáveis pelo grau de dificuldade de passagem do cateter pelo canal cervical.

Não foi observado refluxo de sêmen durante a IAU, o que está de acordo com os resultados encontrados por DALLANORA et al. (2004) e BENNEMANN et al. (2004), os quais utilizaram DIs com volumes de $60 \mathrm{ml}$. No presente estudo, foram infundidos somente $20 \mathrm{ml}$, o que, certamente, favoreceu ainda mais a ausência de refluxo durante a IAU. É possível que a deposição de menor volume de sêmen no amplo ambiente uterino permita a sua rápida distribuição no trato genital da fêmea e, como conseqüência, a nãoocorrência de refluxo. Outra hipótese para explicar a ausência de refluxo de sêmen no momento da inseminação é que com a técnica de IAU há grande distensão da cérvix e do corno uterino pela presença do cateter, induzindo a maior liberação de hormônios envolvidos na contratilidade uterina, tornando o transporte espermático mais eficiente (MARTINEZ et al., 2002). Nas fêmeas inseminadas pela IAT, foi observado refluxo em 10,4\% delas. De acordo com STEVERINK et al. (1998), o refluxo de sêmen na IAT pode ser considerado um processo fisiológico normal, uma vez que é observado na maioria das fêmeas, afetando ou não o desempenho reprodutivo, dependendo do volume refluído.

Na IAU foi observada a presença de sangue na extremidade do cateter ou na espiral da pipeta em 8,4\% (13/154) das fêmeas. Em trabalho recente, DALLANORA et al. (2004) observaram a presença de sangue em 9,5\% das fêmeas. Por outro lado, WATSON \& BEHAN (2002) verificaram sangue em somente 1,8\% das fêmeas. No estudo de DALLANORA et al. (2004), foi avaliada a presença de sangue não só no cateter, mas também no refluxo de sêmen colhido em bolsas de colostomia, até 120 minutos após a IA. Assim, pequenas lesões ocorridas no trato genital da fêmea, que não levariam à presença de sangue no momento da retirada do cateter, poderiam resultar em sangue no refluxo, elevando dessa forma o percentual de fêmeas com a presença de sangue na IAU. No presente experimento, foi realizada inspeção do vestíbulo vaginal à procura de evidências de sangue120 minutos após a IAU. Esses aspectos provavelmente explicam o maior percentual de fêmeas com sangramento, no presente estudo e naquele de DALLANORA et al. (2004), em comparação ao de WATSON \& BEHAN (2002). É possível que determinadas fêmeas possam apresentar lesões préexistentes no trato reprodutivo que predisporiam a traumatismos, principalmente em casos em que foi encontrada maior resistência ao avanço do cateter e maior pressão foi imposta para introduzi-lo além da cérvix (WATSON \& BEHAN, 2002). Para diminuir as possibilidades de traumatismo, o cateter deve ser inserido somente enquanto puder ser manipulado suavemente, não devendo ser forçado.

Não foram observadas diferenças $(\mathrm{P}>0,05)$ na TPr e TPA entre IAU e IAT (Tabela 1), evidenciando que a IAU com 0,5 bilhão de espermatozóides foi eficiente na formação de um reservatório espermático adequado na junção útero-tubárica, capaz de levar a índices de prenhez superiores a 95\%. WATSON \& BEHAN (2002) também não observaram diferenças nas taxas de prenhez e de parto quando compararam a IAU com 1,0 bilhão de espermatozóides e a IAT com 2,0 bilhões de espermatozóides. DALLANORA et al. (2004) não observaram diferenças na TPr e TPA entre IAU e IAT com DIs de 1,5 e 3,0 bilhões de espermatozóides, respectivamente. MEZALIRA et al. (2005), trabalhando com fêmeas inseminadas uma única vez por IAU, abatidas entre 34 e 41 dias de gestação, não encontraram diferença na TPr e no número de embriões utilizando DIs entre 1,0 (84,7\% e 13,3) e 0,5 (85,5\% e 14,3) bilhão de espermatozóides. No entanto, a TPr foi reduzida quando o número de espermatozóides baixou de 0,5 bilhão (85,5\%) para 0,25 bilhão $(77,1 \%)$. WOLKEN et al. (2002) relataram redução da TPr quando foram utilizadas DIs de $20 \mathrm{ml}$ com 0,1 bilhão (65,2\%) de espermatozóides, em comparação às de 0,5 bilhão (77,3\%). Esses trabalhos mostram que, na IAU, doses com menos de 0,5 bilhão de espermatozóides podem comprometer a taxa de prenhez.

A presença de sangue na pipeta de IAU não afetou ( $\mathrm{P}>0,05)$ a TPr (Tabela 2), discordando de DALLANORA et al. (2004), os quais observaram taxa 
Tabela 1 - Taxa de prenhez (TPr), taxa de parto ajustada (TPA) e tamanho da leitegada (TL) de fêmeas suínas inseminadas pela técnica intrauterina (IAU) ou tradicional (IAT).

\begin{tabular}{|c|c|c|c|}
\hline Tratamento & $\operatorname{TPr}\left(\mathrm{n} / \mathrm{n}^{\prime}\right) \%$ & TPA (n/n”) \% & $\mathrm{TL}$ \\
\hline IAU $\left(0,5 \times 10^{9}\right.$ espermatozóides $)$ & $95,4(147 / 154)$ & $92,7(140 / 151)$ & $11,3 \pm 3,4^{\mathrm{a}}$ \\
\hline IAT ( $3,0 \times 10^{9}$ espermatozóides) & $97,9(141 / 144)$ & $95,1(136 / 143)$ & $12,1 \pm 3,4^{b}$ \\
\hline
\end{tabular}

a, b na coluna indicam médias diferentes $(\mathrm{P}<0,05)$.

$\mathrm{n}=$ total de fêmeas prenhes ou paridas.

n'=total de fêmeas inseminadas.

n”=total de fêmeas inseminadas descontando-se as mortas e as descartadas por problemas não-reprodutivos após o diagnóstico de gestação.

de retorno ao estro superior nas fêmeas com sangramento (13,8\%), em comparação à das fêmeas sem sangramento (2,6\%). Embora todas as fêmeas nas quais se verificou sangramento tenham ficado prenhes e parido, a presença de sangue afetou o TL $(\mathrm{P}<0,05)$. As fêmeas da IAU, nas quais o sangue estava presente, apresentaram redução de 2,6 leitões no TL (Tabela 2), o que contribuiu para a redução da média geral das fêmeas submetidas à IAU (Tabela 1). O efeito tóxico do sangue sobre a célula espermática poderia ter sido responsável por uma diminuição da população espermática no ambiente uterino, resultando em menor número de espermatozóides na junção útero-tubárica, que não estariam aptos a fecundar todos os oócitos liberados, explicando o TL reduzido na IAU, em comparação à IAT. Este dado salienta a importância que deve ser atribuída ao treinamento do pessoal que executa a técnica de IAU. Apesar de WATSON \& BEHAN (2002) comentarem que a IAU se trata de uma técnica simples e que, com um mínimo de treinamento, os funcionários da granja podem executá-la, é importante salientar que problemas operacionais poderão comprometer o seu sucesso. No entanto, dados relativos à presença de sangue na IAU e o seu efeito sobre a taxa de prenhez e o número de leitões nascidos ainda são escassos, necessitando de mais avaliações.
O TL diferiu entre os tratamentos $(\mathrm{P}<0,05)$, sendo observada redução de 0,8 leitão nas fêmeas da IAU em comparação à IAT (Tabela 1). Em outros trabalhos, não foram relatadas diferenças no TL, quando a IAU foi efetuada com 1,0 (WATSON \& BEHAN, 2002) ou 1,5 (DALLANORA et al., 2004) bilhão de espermatozóides, em comparação à inseminação tradicional com 3,0 bilhões. MEZALIRA et al. (2005) utilizaram a inseminação intra-uterina com 0,25; 0,5 e 1,0 bilhão de espermatozóides e observaram redução do número de embriões totais somente quando foram utilizados 0,25 bilhão de espermatozóides. A redução do número de espermatozóides por dose na IAU poderia reduzir os índices de fertilidade, caso houvesse problema na qualidade espermática (MEZALIRA et al., 2005). No entanto, no presente estudo, foi utilizado um "pool" de sêmen de reprodutores que se encontravam dentro dos padrões mínimos de qualidade seminal (CBRA, 1998), minimizando um possível efeito negativo dos machos. Dessa forma, outros fatores como intervalo inseminação-ovulação e viabilidade espermática poderiam estar envolvidos na redução do TL. WOLKEN et al. (2002) e MEZALIRA et al. (2005) inseminaram fêmeas com intervalo inseminaçãoovulação de até 24 horas e observaram redução no número de embriões quando foram infundidos menos de 0,5 bilhão de espermatozóides. No presente estudo, as inseminações foram efetuadas a cada 24 horas, sendo

Tabela 2 - Taxa de prenhez (TPr), taxa de parto ajustada (TPA) e tamanho da leitegada (TL) de fêmeas suínas com presença ou ausência de sangue no cateter após a inseminação intra-uterina.

\begin{tabular}{lllr}
\hline Presença de sangue & TPr (n/n') \% & TPA (n/ n”) \% & TL \\
Não & $95,0(134 / 141)$ & $92,0(127 / 138)$ & $11,6 \pm 3,4^{\mathrm{a}}$ \\
Sim & $100,0(13 / 13)$ & $100,0(13 / 13)$ & $9,0 \pm 3,3^{\mathrm{b}}$ \\
\hline
\end{tabular}

a, b na coluna indicam médias diferentes $(\mathrm{P}<0,05)$.

$\mathrm{n}=$ total de fêmeas prenhes ou paridas.

n'=total de fêmeas inseminadas.

n” =total de fêmeas inseminadas descontando-se as mortas e as descartadas por problemas não-reprodutivos após o diagnóstico de gestação.

Ciência Rural, v.37, n.6, nov-dez, 2007. 
possível que esse intervalo seja crítico, quando se trata de doses com 0,5 bilhão de espermatozóides ou menos, em termos de manutenção de uma população espermática compatível com altas taxas de fecundação. No entanto, esse aspecto necessita de mais estudos para o seu esclarecimento.

\section{CONCLUSÃO}

A taxa de prenhez e a taxa de parto ajustada não diferiram empregando-se a inseminação artificial tradicional com 3 bilhões de espermatozóides por dose ou a inseminação artificial intra-uterina com 0,5 bilhão de espermatozóides. Entretanto, na deposição uterina, observou-se uma redução no tamanho da leitegada. Com isso, o número mínimo de espermatozóides utilizados com a inseminação artificial intra-uterina, para a obtenção de índices semelhantes aos alcançados na inseminação tradicional, deve ser superior a 0,5 bilhão.

\section{REFERÊNCIAS}

BAKER, R.D. et al. Effect of volume of semen, number of sperm and drugs on transport of sperm in artificially inseminated gilts. Journal of Animal Science, v.27, p.88-93, 1968.

BENNEMANN, P.E. et al. Reproductive performance of sows submitted to intrauterine insemination in different preovulatory intervals. Animal Reproduction. v.1, p.106-110, 2004

BENNEMANN, P.E. et al. Artificial insemination of gilts with different semen storage periods associated to different preovulatory intervals. Reproduction in Domestic Animals, v.40, p.507-510, 2005.

CBRA (COLÉGIO BRASILEIRO DE REPRODUÇÃO ANIMAL). Manual para exame andrológico e avaliação de sêmen animal. 2.ed. Belo Horizonte: CBRA, 1998. 49p.
DALLANORA, D. et al. Desempenho reprodutivo de fêmeas suínas inseminadas pela técnica intra-uterina ou tradicional. Pesquisa Agropecuária Brasileira, v.39, p.815-819, 2004.

HANCOCK, J.L. Pig insemination technique. Veterinary Record, v.71, p.527, 1959.

HANCOCK, J.L.; HOVELL, J.R. The effect of semen volume and number of spermatozoa on the fertility of intra-uterine inseminations of pigs. Animal Production, v.3, p.153-160, 1961.

MARTINEZ, E.A. et al. Deep intrauterine insemination and embryo transfer in pigs. Journal of Reproduction and Fertility, Supplement 58, Control of Pig Reproduction VI, p.301-311, 2001.

MARTINEZ, E.A. et al. Minimum number of spermatozoa required for normal fertility after deep intrauterine insemination in non-sedated sows. Reproduction, v.123, p.163-170, 2002.

MEZALIRA, A. et al. Influence of sperm cell dose and postinsemination backflow on reproductive performance of intrauterine inseminated sows. Reproduction in Domestic Animals, v.40, p.1-5, 2005.

RATH, D. et al. Low dose insemination technique in the pig. In: BOAR SEMEN PRESERVATION CONGRESS, 4., 2000, Beltsville. Proceedings... Beltsville, USA: Allen, 2000. p.115118.

SAS INSTITUTE. SAS/STAT, User's guide. Version 8. Cary, NC, 1999. 1464p.

STEVERINK, D.W. et al. Semen backflow after insemination and its effect on fertilization results in sows. Animal Reproduction Science, v.54, p.109-119, 1998.

WATSON, P.F.; BEHAN, J.R. Intrauterine insemination of sows with reduced sperm numbers: results of a based field trial. Theriogenology, v.57, p.1683-1693, 2002.

WOLKEN, A. et al. Sows can successfully be inseminated nonsurgically into the distal uterine horn with a highly reduced number of sperm cells. Theriogenology, v.57, n.1, p.392, 2002. 